\title{
Diagnostic Significance of Distal Compound Muscle Action Potential (CMAP) Duration-A Case Report
}

\author{
Edvard Ehler ${ }^{1,2}$, Nela Kopecka ${ }^{1}$ and Petra Mandysova ${ }^{1,2}$ \\ ${ }^{1}$ Department of Neurology, Pardubice Regional Hospital, Pardubice, Czech Republic \\ ${ }^{2}$ Faculty of Health Studies, University of Pardubice, Pardubice, Czech Republic
}

Corresponding author: Edvard Ehler, Department of Neurology, Regional Hospital, 44 Kyjevska, Pardubice, 532 03, Czech Republic; Tel; 00420 466014701; Fax: 00420 466014702; E-mail: edvard.ehler@nempk.cz

Received: Mar 02, 2016; Accepted: Mar 29, 2016; Published: Apr 04, 2016

\section{Abstract}

The diagnostic criteria of chronic inflammatory demyelinating polyradiculoneuropathy (CIDP) are based on clinical findings and auxiliary investigations, of which the most important ones are neurophysiological findings. Since the European Federation of Neurological Societies/ Peripheral Nerve Society (EFNS/PNS) incorporated distal CMAP duration into the neurophysiological diagnostic criteria, their sensitivity increased to $81.3 \%$ and specificity to $96.2 \%$. We describe a case of a 58 -year-old man who was referred to our electromyography (EMG) laboratory for a second opinion regarding his diagnosis. The patient exhibited sensory-motor polyneuropathy with prominent hypotrophy of the calf muscles. Electrodiagnostic testing prior to admission had revealed a probable axonal neuropathy. In our EMG laboratory, we accomplished nerve conduction studies (NCS) of both hands and legs and we noted a prominent increase of distal CMAP duration, whereas distal motor latencies (DML) were within normal limits. As the CMAP amplitude in the tibial and peroneal nerves was less than 1 millivolt $(\mathrm{mV})$, the existence of myelinopathy could not be inferred from any other parameters identified in the lower extremities. Neurophysiological investigation of both hands was necessary, even though the clinical picture of polyneuropathy was substantially more prominent in the legs. It is important to distinguish between axonal and myelin sheath lesions in order to understand the pathogenetic aspects of neuropathy. In addition, treatment of demyelinating autoimmune neuropathies can be offered and it is effective. In our patient, distal CMAP duration was the main discriminating parameter among the various neurophysiological parameters of the CIDP diagnostic criteria.

Keywords: CMAP duration; Motor conduction studies; Immunosuppressive therapy; Neurophysiological criteria for CIDP

\section{Introduction}

The diagnostic criteria of CIDP are based on clinical findings and auxiliary investigations. The ones of greatest importance are the neurophysiological criteria, originally determined by the American Academy of Neurology (AAN) and later, in 2010, by the EFNS/PNS. The earlier established parameters included motor conduction velocity, distal latency prolongation, F-wave latency prolongation, conduction block, and temporal dispersion. The EFNS/PNS added a new parameter - distal CMAP duration. A multicentre study established a sensitivity level of only $63 \%$ and a specificity of $99.3 \%$ for the AAN criteria, whereas sensitivity and specificity levels of $81.3 \%$ and $96.2 \%$, respectively, were reached for the EFNS/PNS criteria [1]. Prolonged distal CMAP duration is of high importance; a value equal to or greater than 9 milliseconds (ms) in only one of four nerves distinguishes CIDP from other neuropathies with a sensitivity of 0.78 and specificity of 0.94 [2].

We describe a patient with a one-year history of paraesthesia and weakness in all 4 limbs, whose prolonged distal CMAP duration was the decisive parameter for the diagnosis of CIDP.

\section{Case report}

We describe a case of a 58-year-old man with hypertension who had undergone aortic valve replacement, necessitating long-term anticoagulant therapy; he had been engaging in sports. He has absolved ground school; he is a postman and driver of postal car. He is non- smoker and only exceptionally drinks a glass or two of beer. The patient initially presented with a one-year history of paraesthesia and hypaesthesia of the feet and hands along with hypotrophy of small muscles of the feet and calves. There were decreased reflexes C7, C8 and L2-4 and non-elicitable reflexes L5-S2. Mild hypaesthesia on the distal phalangs of all fingers with clumsy pinch and difficulties with skilled movements (buttoning). We have found hypaesthesia distally of patella with loss of vibration sense on feet. He was able to squat down only with the help of arms and standing on one leg disclosed pelvis instability. Nonetheless, he was still able to walk on his toes and heels.

The patient was referred for a second opinion regarding his diagnosis of distal sensory-motor polyneuropathy of axonal type. Electrodiagnostic testing prior to admission had revealed very low CMAP amplitude in the lower extremities, and mild slowing of motor conduction velocities (MCV) and sensory conduction velocities (SCV). By means of a needle EMG, a severe chronic denervation syndrome in the muscles of the lower legs had been ascertained. 
In our EMG laboratory, we performed nerve conduction studies (NCS) of both hands and legs, which showed a prominent increase of distal CMAP duration, whereas distal motor latencies (DML) were within normal limits. Sensory nerve action potentials (SNAP) in the lower and upper extremities were acquired; however, low amplitude and low sensory conduction velocity $(25-35 \mathrm{~m} / \mathrm{s})$ were observed (Table 1), (Figures 1-3).

Table 1 Motor nerve conduction studies

\begin{tabular}{|c|c|c|c|c|c|}
\hline Nerve (+muscle) & $\begin{array}{l}\text { Distal motor } \\
\text { latency (ms) }\end{array}$ & CMAP amplitude (distal/proximal,mV) & $\begin{array}{l}\text { Distal CMAP } \\
\text { duration }(\mathrm{ms})\end{array}$ & $\begin{array}{l}\text { Conduction velocity } \\
(\mathrm{m} / \mathrm{s})\end{array}$ & $\begin{array}{l}\text { F-wave latency } \\
\text { (ms) }\end{array}$ \\
\hline R Median (APB) & 4.35 & $12.0 / 10.6$ & 7.20 & 47.6 & 32.4 \\
\hline L Median (APB) & 4.30 & $13.0 / 13.0$ & 8.35 & 49.5 & 31.5 \\
\hline R Ulnar (ADM) & 3.70 & $6.8 / 5.9 / 5.4$ & 7.45 & $53.0 / 40.0$ & 33.1 \\
\hline L Ulnar (ADM) & 3.90 & $6.3 / 6.1 / 5.7$ & 9.22 & $49.5 / 43.6$ & 34.5 \\
\hline R peroneal (EDB) & 5.35 & $0.7 / 0.7$ & 5.6 & 35.6 & 0 \\
\hline R Tibial $(\mathrm{AH})$ & 6.40 & $0.3 / 0.1$ & 6.15 & 37.1 & 63.7 \\
\hline R Femoral (VL) & 5.25 & 4.3 & 13.95 & & \\
\hline
\end{tabular}
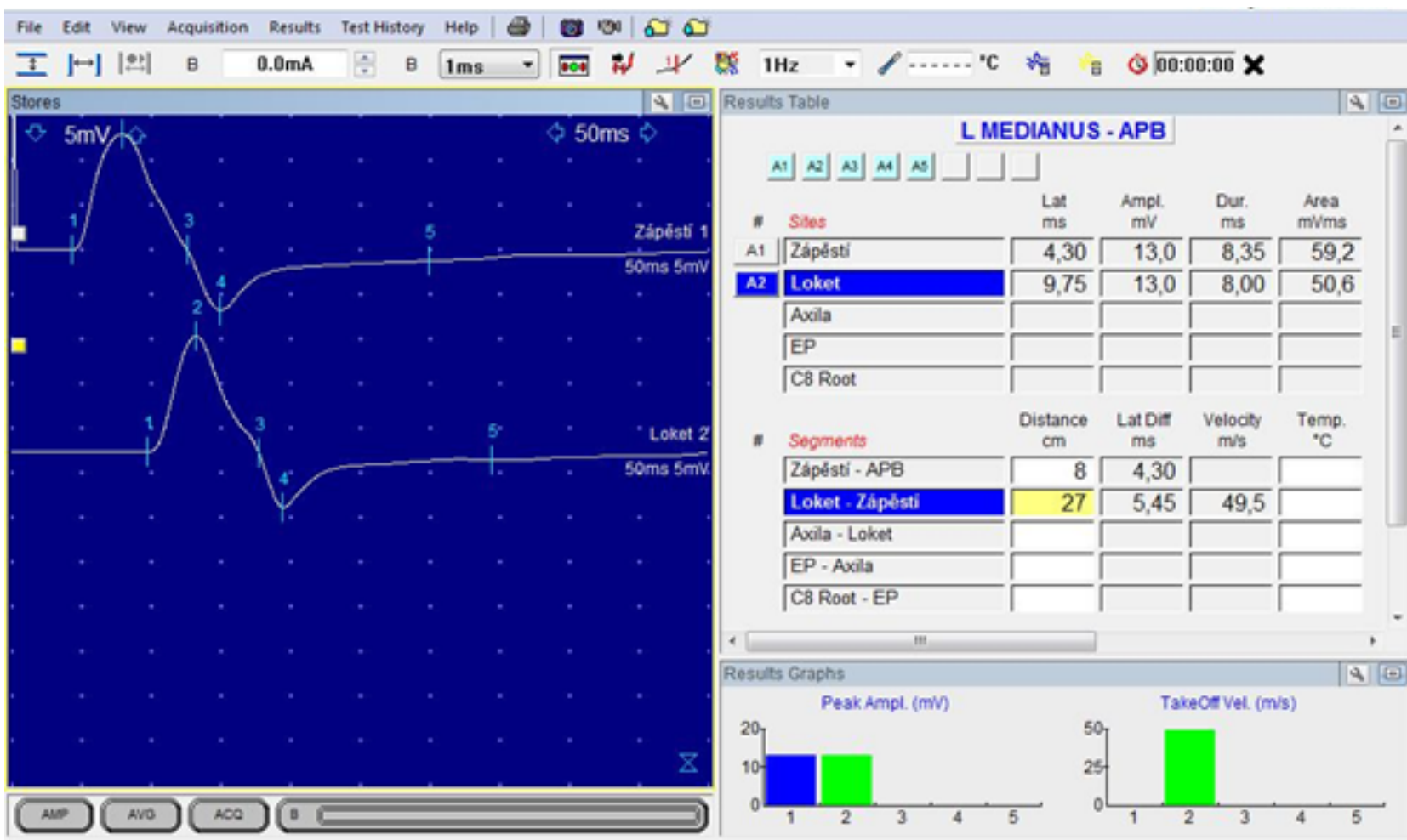

Figure 1 Motor conduction studies of median nerve - before therapy

Lumbar puncture findings were normal, antibodies against gangliosides were not detected, and oncological screening was negative.

Based on the neurophysiological findings we concluded that the EFNS/PNS criteria for the diagnosis of CIDP were fulfilled. The most important parameter was the duration of distal CMAP of the median and ulnar nerves bilaterally. Because the DML and MCV were within normal range, the demyelination changes were probably located in the distal forearms and wrists. The CMAP amplitude in the tibial and peroneal nerves was less than $1 \mathrm{mV}$; therefore, the existence of myelinopathy could not be inferred from any other parameters identified in the lower extremities.

An intravenous infusion of methylprednisolone (1 g administered 5 times) led to substantial relief of neuropathic pain and improved walking ability (Figure 4). Currently, the patient is on maintenance immunosuppression with prednisone $10 \mathrm{mg}$ every other day and azathioprine $100 \mathrm{mg}$ once daily. 

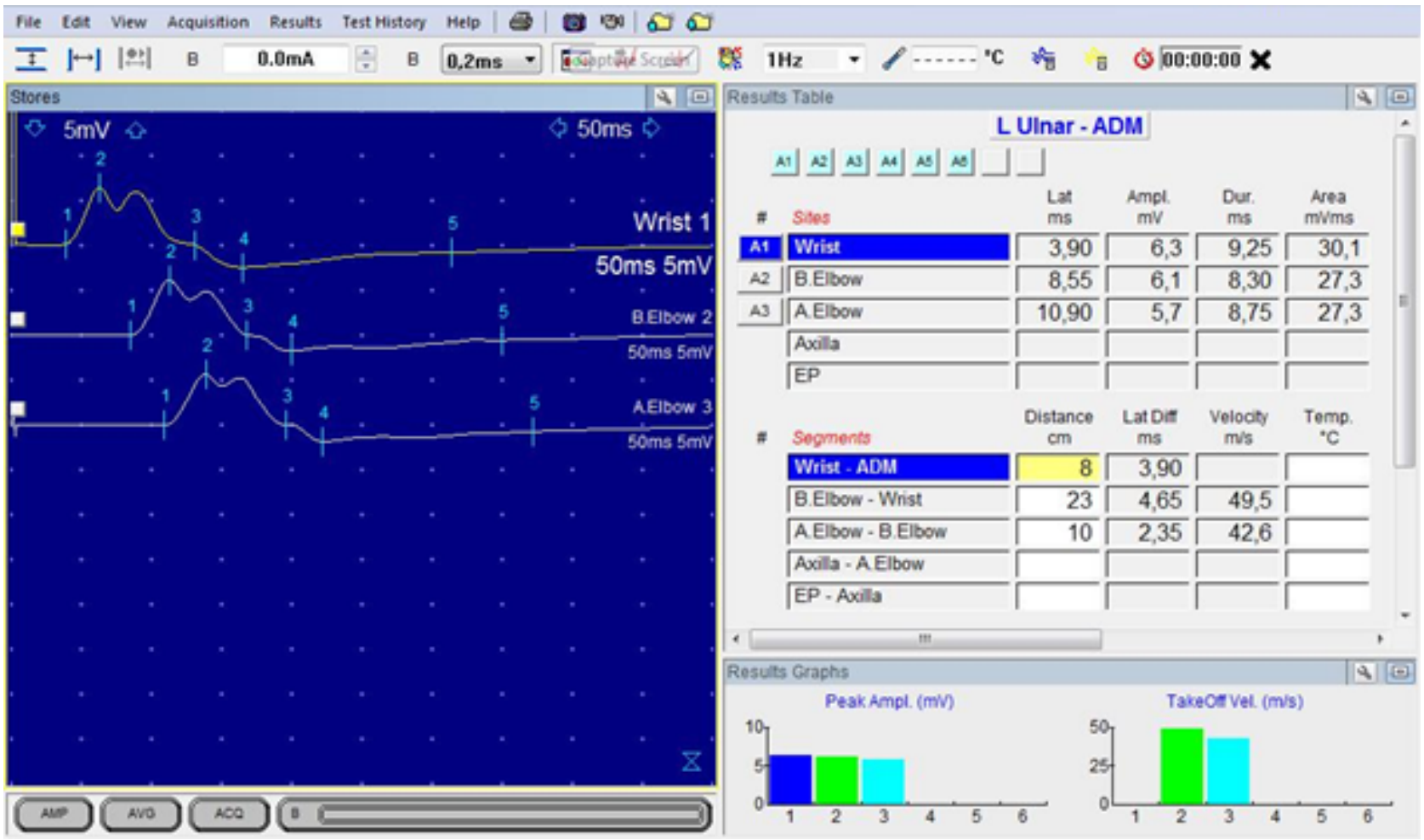

Figure 2 Motor conduction studies of ulnar nerve - before therapy

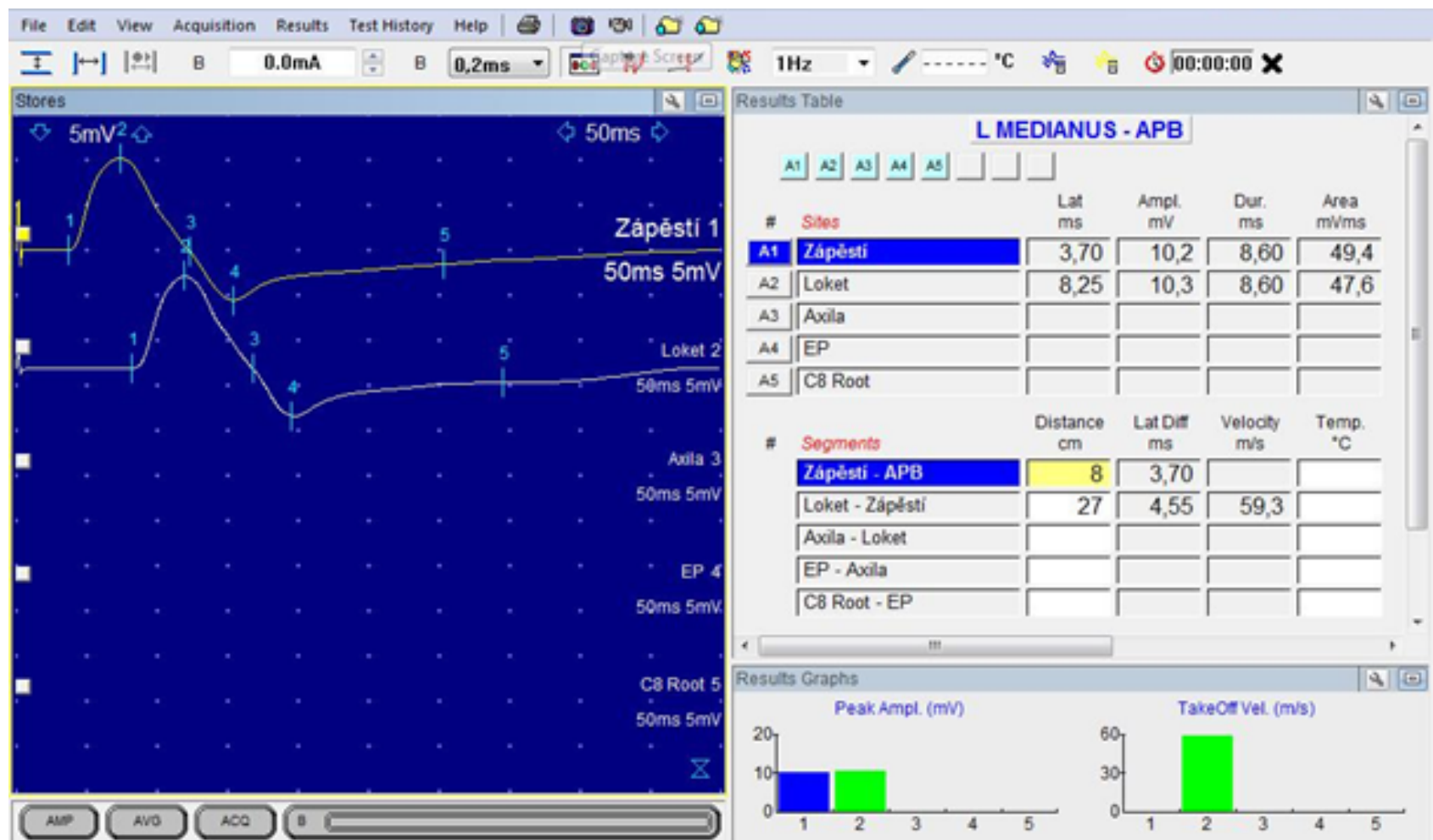

Figure 3 Motor conduction studies of median nerve - after methylprednisolone therapy. Changes: distal motor latency is shorter (-0.60 ms), motor conduction velocity slightly decreased, duration of distal CMAP insignificantly longer (+ $0.25 \mathrm{~ms})$ 

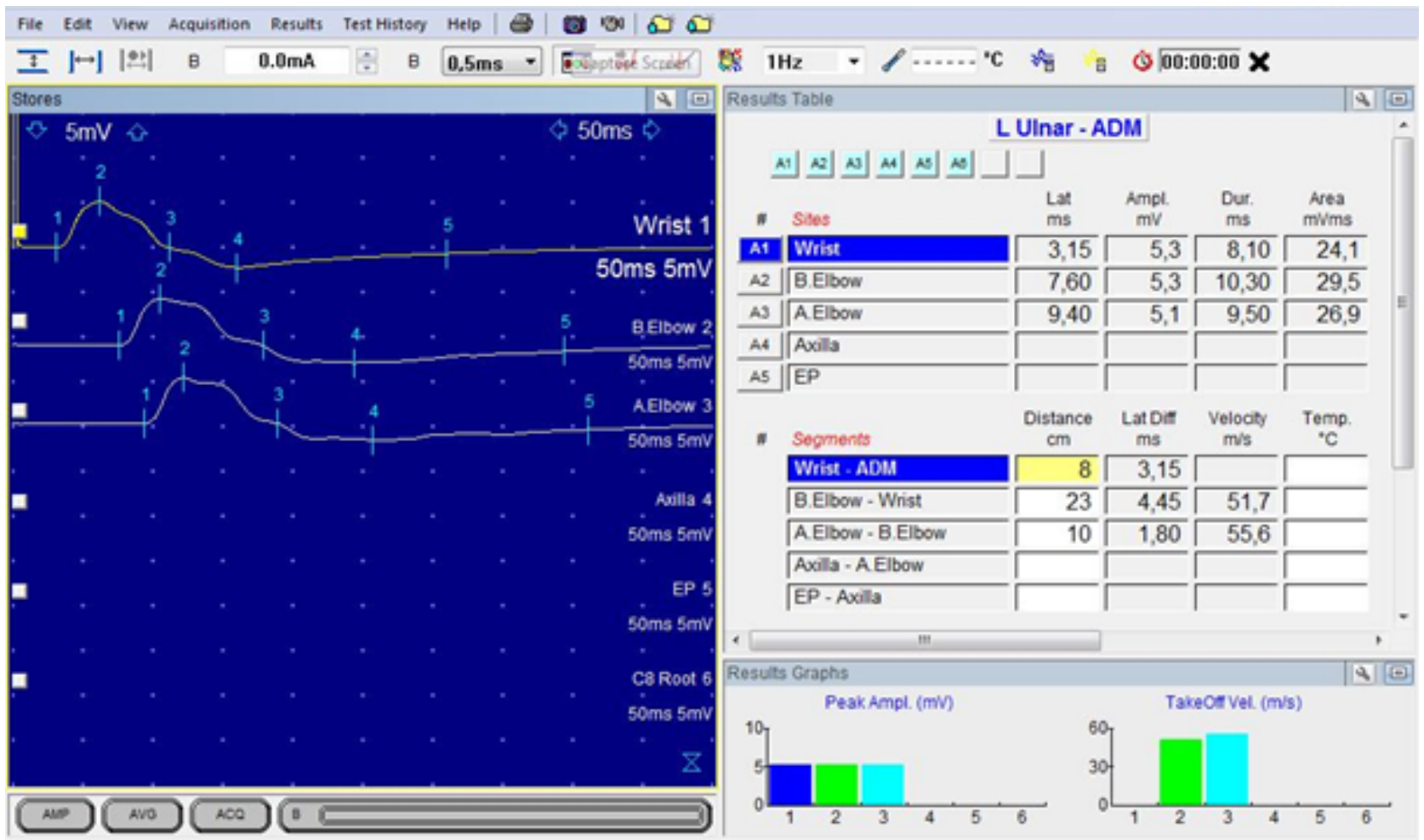

Figure 4 Motor conduction studies of ulnar nerve - after methylprednisolone therapy. Changes: distal motor latency is shorter (-0.75 ms), motor conduction velocity slightly increased, duration of distal CMAP shorter (-1.15 ms)

\section{Discussion}

Diagnosis of CIDP requires that a patient has a chronic nongenetic polyneuropathy, progressive for at least eight weeks, without a serum paraprotein. In typical CIDP, there is a symmetrical onset of motor symptoms, symmetrical weakness of four limbs, and proximal weakness in at least 1 limb. A recordable CMAP should be in at least $75 \%$ of motor nerves with either prolonged DML (in $>50 \%$ of 2 nerves) or abnormal MCV (in $>50 \%$ of 2 nerves) or abnormal F-wave latency (in $>30$ $\%$ of 2 nerves) [3].

Thaisetthawatkul et al. aimed to assess distal CMAP duration as a diagnostic criterion for CIDP. Prolonged duration ( $\geq 9 \mathrm{~ms}$ ) enabled to distinguish CIDP from diabetic neuropathy, ALS, and musculoskeletal pain syndrome [2]. The neurophysiological parameters were further specified in the EFNS/PNS criteria, which were amended to include distal CMAP duration. CMAP duration is a useful index to detect demyelination in the distal nerve segments. To provide widelyavailable reference data of CMAP duration, a JapaneseEuropean multicentre prospective study aimed to use different low frequency filters [4]. Their results showed that distal CMAP duration is largely dependent on low frequency filter settings. The cut-off values for a low frequency filter of $20 \mathrm{~Hz}$ were $7.4 \mathrm{~ms}$ for the median nerve, $7.8 \mathrm{~ms}$ for the ulnar nerve, $8.1 \mathrm{~ms}$ for the peroneal nerve, and 8.0 for the tibial nerve [4].

In our patient, we found that distal CMAP duration was markedly prolonged $(7.10 \mathrm{~ms}$ and $8.60 \mathrm{~ms}$ in the median nerves and $7.45 \mathrm{~ms}$ and $9.22 \mathrm{~ms}$ in the ulnar nerves). In three of the four nerves, distal CMAP duration was prolonged, yet in both hands, the DML values were within normal range. Normal DML means that there is no demyelination in the terminal motor nerves. Based on the pattern of breakdown of the blood-nerve barrier, we believe that the myelin sheath lesions were in the intermediate of nerves, from forearms to the wrists [5].

\section{Conclusion}

Although the clinical picture of distal sensory-motor polyneuropathy resembles that of axonal neuropathy and predominantly affects the legs, EMG investigation needs to include not only the legs but also both hands. In cases of low CMAP amplitude in the lower extremities, it is not possible to infer the diagnosis from an EMG of the legs and to distinguish axonal lesions from myelin sheath lesions. In such cases, an evaluation of distal CMAP duration of the median and ulnar nerves is the most important and the main discriminating parameter.

\section{Conflict of Interest/Disclosures}

The authors declare that they have no financial or other conflicts of interest in relation to this research and its publication. 


\section{References}

1. Van den Bergh PY, Rajabally YA (2013) Chronic inflammatory demyelinating polyradiculoneuropathy. Presse Med 42: 203-215.

2. Thaisetthawatkul P, Logigian EL, Herrmann DN (2002) Dispersion of the distal compound muscle action potential as a diagnostic criterion for chronic inflammatory demyelinating polyneuropathy. Neurology 59: 1526-1532.

3. Koski CL, Baumgarten M, Magder LS, Barohn RJ, Goldstein J, et al. (2009) Derivation and validation of diagnostic criteria for chronic inflammatory demyelinating polyneuropathy. J Neurol Sci 277: 1-8.
4. Mitsuma S, Van den Bergh P, Rajabally YA, Van Parijs V, MartinLamb D, et al. (2015) Effects of low frequency filtering on distal compound muscle action potential duration for diagnosis of CIDP: A Japanese-European multicentre prospective study. The Tokyo Metropolitan Neuromuscular Electrodiagnosis Study Group. Clin Neurophysiol 126: 1805-1810.

5. Shimizu F, Sawai S, Sano Y, Beppu M, Misawa S, et al. (2014) Severity and patterns of blood-nerve barrier breakdown in patients with chronic inflammatory demyelinating polyradiculoneuropathy: correlations with clinical subtypes. PloS One 9: e104205. 